\title{
Reflexões sobre o Ensino de Bioética e Cuidados Paliativos nas Escolas Médicas do Estado de Minas Gerais, Brasil
}

\author{
Reflections on the Teaching of Bioethics and \\ Palliative Care across Medical Schools in the \\ State of Minas Gerais, Brazil
}

\section{PALAVRAS-CHAVE}

- Ética;

- Bioética;

- Cuidados Paliativos;

- Educação Médica.

\section{KEYWORDS}

- Ethics;

- Bioethics;

- Palliative Care;

- Medical Education.

Recebido em: 28/06/2015

Reencaminhado em: 17/12/2015

Aprovado em: 18/01/2016

REVISTA BRASILEIRA DE EDUCAÇĀO MÉDICA
José Ricardo de Oliveira ${ }^{I}$ Amauri Carlos Ferreira ${ }^{I I}$ Nilton Alves de Rezendel Letícia Pereira de Castro ${ }^{I I I}$

\section{RESUMO}

Este artigo analisa a atual situação do ensino de bioética e cuidados paliativos nas escolas médicas do Estado de Minas Gerais, Brasil. O ensino de cuidados paliativos na graduação é importante para se compreender a relação escola-médico-equipe interdisciplinar de assistência à saúde no que diz respeito à terminalidade da vida humana. $O$ estudo exploratório de caso e de natureza qualitativa utilizou três instrumentos operacionais: questionário, entrevista e documento informatizado. Foram estudadas 28 escolas médicas para se verificar como é realizado o ensino de bioética e cuidados paliativos. Foi também aplicado questionário a professores para avaliar o ensino desta temática, além de trabalho de campo, com aplicação de questionário estruturado a um grupo de alunos, em duas destas escolas, para aprofundamento do estudo, em dois momentos distintos da formação dos alunos, o que permitiu uma discussão crítica e transversal da formação acadêmica. Os resultados mostraram que o conhecimento de bioética com ênfase em cuidados paliativos e com a formação de médicos que atendam às necessidades emergentes desta área da saúde não está suficientemente contemplado nas escolas médicas do Estado de Minas Gerais, assim como não se observa uma correlação entre o ensino de bioética e o ensino de cuidados paliativos. Propõe-se uma reflexão e uma ação programática para a formação docente e subsequente inserção curricular específica e de caráter longitudinal do ensino de bioética e cuidados paliativos nos cursos de graduação das escolas médicas de Minas Gerais.

\section{ABSTRACT}

This paper reviews the current status of the teaching of bioethics and palliative care in medical schools across the state of Minas Gerais, Brazil. The teaching of palliative care in the state's medical courses is paramount to students' understanding of the medicine-physician-interdisciplinary healthcare team's handling of the terminal nature of human life. A case study of a qualitative nature was employed in order to analyze 28 medical schools registered by the Ministry of Education and Culture, with structured interviews conducted in the aim of identifying how palliative care is taught across the medical courses. Field work was therefore conducted via these structured interviews held with students in two medical schools. Thereafter, students in their final year of the medical course and on hospital-school internships in two medical schools were interviewed. The results show that those responsible for the curricula are unconcerned about knowledge dissemination in bioethics, with their focus instead on palliative care and on the training of physicians to provide support to emergent needs in this field of science. Considering the lack of concern over the teaching of bioethics with a focus on palliative care in the medical schools' formal curricula, we propose a reflection and programmatic action on the training of teachers in this field of knowledge, as well as the insertion of palliative care teaching into the schools' degree curricula.

\footnotetext{
Universidade Federal de Minas Gerais, Belo Horizonte, MG, Brasil.

II. Pontifícia Universidade Católica de Minas Gerais, Belo Horizonte, MG, Brasil.

III. Hospital Municipal de São José dos Campos, São José dos Campos, SP Brasil.
} 


\section{INTRODUÇÃO}

A pergunta que se faz é: como cuidar dessas pessoas que estudam em salas de anatomia onde jazem cadáveres desconhecidos, mutilam pequenos animais em laboratórios de fisiologia, veem corpos vivos sendo abertos em centros cirúrgicos, observam a dor e a tristeza fechados em hospitais e frequentam toda sorte de ambientes onde o sofrimento humano chega ao limite suportável para a vida? ${ }^{1}$ (p. 159)

A assistência aos pacientes portadores de doenças terminais estabelece uma relação de cuidado entre os membros da equipe interdisciplinar de saúde e pacientes, familiares e cuidadores. Mas qual é o papel desempenhado por essas pessoas durante o processo do morrer e da morte?

A formação e a capacitação do futuro médico nos aspectos ético, bioético e paliativista, que visam garantir sua excelência profissional, são importantes no cenário da atenção aos pacientes com doenças em fase terminal. Neste sentido, esta pesquisa contempla a tríade escola de medicina-médico-equipe interdisciplinar de assistência à saúde. Entretanto, os responsáveis pela elaboração dos currículos nas escolas de Medicina não estão muito preocupados, ao que parece, com a formação de profissionais que atendam às necessidades emergentes do campo da bioética com ênfase em cuidados paliativos (CP).

Este aspecto é relevante se for considerado que o Brasil está em processo de transição demográfica e epidemiológica. O País está passando para a fase de predominância das doenças crônico-degenerativas, em que existe limitação para as atividades de vida diária e intensificação de cuidados que, muitas vezes, ao contrário do que se espera, comprometem a qualidade de morte. Em 2006, faleceram 1.031.691 pessoas no Brasil ${ }^{2}$. Apenas os óbitos decorrentes de doenças de evolução crônica ou degenerativa e neoplasias corresponderam ao montante de mais de 725 mil brasileiros ${ }^{2}$, com possibilidade de intenso sofrimento e alto custo financeiro em decorrência da falta de formação, educação continuada e recursos humanos direcionados ao CP.

Nos países desenvolvidos, a tendência é reduzir as internações hospitalares e incentivar o acompanhamento do paciente no domicílio. Também no Brasil o modelo de atenção domiciliar deverá adquirir importância estratégica, tanto na saúde pública como na saúde suplementar. Portanto, é necessário investir na formação de recursos humanos e difundir os conhecimentos demográficos e epidemiológicos da população brasileira para divulgar o melhor grau das opções assistenciais ao final da vida ${ }^{3,4}$.
Desde a segunda metade do século XX, ocorre a reflexão-ação bioética e a prática na atenção a pacientes com doença avançada e terminal, e o CP emerge como entidade assistencial em alguns países. O processo do morrer com dignidade, a questão da bioética e do CP e a prática de trabalho em equipe interdisciplinar de saúde revigoram a tendência do ensino curricular de bioética e de CP nas escolas médicas (EM) internacionais, marcado com o conceito de doença e de sua "cura anatômica" como principal função do médico. Neste aspecto, "[...] o tema levanta questões sobre o sentido da vida, o objetivo do tratamento médico, o direito da pessoa para determinar quando o tratamento e a sua vida devem terminar." ${ }^{15}$ (p. 127, tradução livre).

Esta reflexão parte do pressuposto vivenciado pelos autores de que é preciso implantar o ensino de CP nas EM para melhor atuação interdisciplinar e o cuidado do paciente portador de doença avançada e terminal. Baseia-se no corpus teórico de filósofos, bioeticistas, sociólogos, pesquisadores de CP e de educação médica. Esta pesquisa privilegia dois campos do conhecimento: o da bioética e o do CP.

Há rica literatura e práxis, sedimentada internacionalmente, sobre a bioética e o CP. Entretanto, no Brasil, convive-se com dilemas éticos relativos ao tema, tanto no modelo do ensino médico, quanto na qualidade de assistência ao processo de morrer com dignidade ${ }^{6}$. Mas o relato provocativo de que “Não existe ensino de cuidados paliativos no Brasil. Os médicos brasileiros não são treinados para lidar com pacientes portadores de doenças terminais. ${ }^{\prime 7}$ (p. 19, tradução livre) enfatiza o olhar pedagógico descompromissado das EM sobre esta questão $^{8}$. Essa realidade do ensino de CP nas EM coincide com os dados de trabalhos no campo da assistência deficitária de $\mathrm{CP}$ oncológicos no Brasil ${ }^{3,4}$. O estudo intitulado "A Qualidade da Morte" mostra que, em um ranking de 40 países, no quesito "assistência na fase final de vida", o Brasil ocupa o 38ํㅣㅁ lugar, ficando à frente apenas de Uganda (39ํ) e da Índia $\left(40^{\circ}\right)^{9}$.

O objetivo geral deste estudo foi analisar os currículos das EM do Estado de Minas Gerais (EMMG), com a finalidade de verificar como está sendo aplicado o ensino de CP na graduação.

$\mathrm{Na}$ tentativa de entender e responder a esses questionamentos, o texto foi estruturado em três seções: análise do ensino de bioética e cuidados paliativos nas escolas médicas; discurso programático; reflexões e conclusões.

\section{ENSINO DE BIOÉTICA E CUIDADOS PALIATIVOS NAS EMMG: METODOLOGIA}

Para verificar como se encontra o ensino de bioética e CP nas EMMG, foi realizado um estudo exploratório de caso e de natureza qualitativa, que utilizou o trabalho de campo e três ins- 
trumentos operacionais: questionário estruturado, entrevista e documento informatizado. O projeto foi divulgado por meio de reuniões com a coordenação de quatro cursos de Medicina localizados na cidade de Belo Horizonte e do envio de documentação em formato eletrônico às demais escolas.

Em 2011, o Estado de Minas Gerais ocupava o segundo lugar no Brasil em número de EM e vagas para o primeiro ano do curso médico, totalizando 32 faculdades e 2.750 vagas $^{10,11}$. Dessas escolas, 11 (34\%) são escolas públicas e 21 (66\%) escolas privadas. Essa distribuição condiz com o que se observou no restante do Brasil, onde $44 \%$ são escolas públicas e 56\% escolas privadas $^{10}$.

Para a pesquisa, foi utilizado um questionário híbrido da tipologia de escolas médicas e da dimensão ética do processo de formação, de acordo com Lampert ${ }^{12}$ e Finkler ${ }^{13}$. Já a abordagem de bioética e $\mathrm{CP}$ foi acrescida junto ao questionário de Finkler ${ }^{13}$.

O trabalho foi desenvolvido em três etapas (Figura 1):

- Primeira etapa: a amostra foi composta por 32 EMMG autorizadas pelo $\mathrm{MEC}^{11}$. Quatro escolas foram excluídas: uma em decorrência de seu fechamento, e outras três por terem sido criadas em datas posteriores ao início da pesquisa. Das $28 \mathrm{EM}$, seis foram selecionadas para o estudo piloto, utilizando critérios intencionais por possuírem registros curriculares do objeto de pesquisa. Uma escola não consentiu em participar, ficando a amostra constituída de cinco escolas (denominadas EM1 a EM5) para este estudo piloto;

- Segunda etapa: trabalho de campo com visitas técnicas e aplicação de questionário estruturado aos alunos, em duas EMMG (EM1 e EM2), sem e com ensino curricular de bioética e $C P$, respectivamente;

- Terceira etapa: trabalho de campo, com visitas técnicas e realização de entrevistas com um grupo de alunos que cursava o último ano e internato no Hospital-Escola (HE), em duas EMMG (EM1 e EM2).

Portanto, os questionários foram aplicados em três etapas. Na primeira, foi aplicado um questionário estruturado a cinco EM. Ao final dessa etapa, foram consultadas as demais 23 EMMG, com a aplicação eletrônica do questionário estruturado de pesquisa, mas elas não responderam à enquete. $\mathrm{Na}$ segunda e terceira etapas, a pesquisa de campo se desdobrou em entrevistas, anotações no caderno de campo, análise documental e aplicação de questionário estruturado a um grupo de alunos de graduação de duas dessas 28 EMMG, para subsidiar uma discussão crítica e longitudinal em relação ao ensino acadêmico de CP.
Podem-se identificar, nesta pesquisa, pelo menos dois pontos de estrangulamento e limitações. Um dos objetivos era atingir o universo de 28 EM em Minas Gerais. Todavia, não foi possível obter o resultado esperado. Cinco EM responderam aos questionários. Os demais dados, referentes às matrizes curriculares, foram coletados em rede informatizada. É também possível que haja limitações conceituais e escassez de marcos teóricos, apesar da referência de autores balizados que contribuíram na interpretação e análise dos dados.

Figura 1

Fluxograma das três etapas

metodológicas (coleta de dados)

1ํㅡㄹ ETAPA 2믈 ETA

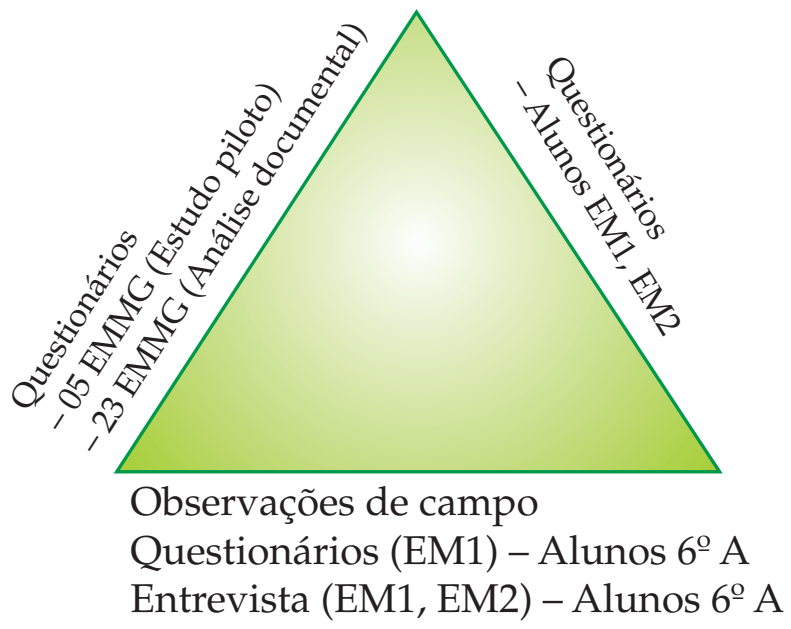

3모A ETA

\section{ANÁLISE DE QUESTIONÁRIOS APLICADOS ÀS EMMG E DADOS DOCUMENTAIS}

A EM1 possui tipologia tradicional no enfoque teórico, atividades de ética e de bioética pontuais e nenhuma abordagem pedagógica em CP. A EM2 e a EM3 possuem tipologia inovadora no enfoque teórico e disciplinas ou estratégias educacionais curriculares direcionadas para ética, bioética e CP. A EM4 foi considerada de tipologia tradicional quanto ao enfoque teórico, mas apresentava abordagem pedagógica em CP. As EM possuíam disciplinas de ética em níveis diferentes (Quadro 1). 


\begin{tabular}{|c|c|c|c|c|c|}
\hline \multicolumn{6}{|c|}{ Quadro 1} \\
\hline \multicolumn{2}{|c|}{ Categorias/Unidades temáticas } & Tradicional & Inovadora & Avançada & Não respondeu ( ${ }^{(1)}$ \\
\hline \multirow{6}{*}{ 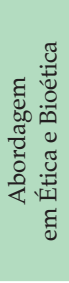 } & Concepção de ética &.. & EM1 & EM2, EM3, EM5 & EM4 \\
\hline & Ética docente &.. & EM4 & EM2, EM3, EM5 & EM1 \\
\hline & Inserção da Ética na matriz curricular &.. & EM1, EM3, EM4 & EM2, EM5 & .. \\
\hline & Disciplinas de Ética & EM1 & EM4, EM5 & EM2, EM3 & \\
\hline & Formação docente &.. & EM1, EM2, EM3, EM5 &.. & EM4 \\
\hline & Referenciais teóricos em Bioética &.. & EM1 & EM2, EM3, EM4, EM5 &.. \\
\hline \multirow{6}{*}{ 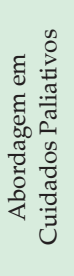 } & Concepção & EM4 & .. & EM2, EM3, EM5 & EM1 \\
\hline & Inserção curricular &. & EM1, EM2, EM3, EM4 & EM5 &.. \\
\hline & Disciplina & EM4, EM5 & EM2, EM3 & .. & EM1 \\
\hline & Formação docente & EM2 & EM4, EM5 & EM3 & EM1 \\
\hline & Referenciais teóricos & EM1 & EM3, EM5 & EM2 & EM4 \\
\hline & Barreiras & EM1, EM4 &.. &.. & EM2, EM3, EM5 \\
\hline
\end{tabular}

(1) Não respondeu = não disponibilizou o dado.

Fonte: Dados da pesquisa.

Em síntese, os estudos brasileiros, publicados nas décadas de 1980, 1990 e 2000, mostram que o espaço curricular para o ensino da ética médica permanece desorganizado e com variedade de programas e que a inserção de bioética nas EM foi tardia em relação à ética médica ${ }^{14,15}$. Observa-se ainda a pequena carga horária destinada ao ensino de ética (inferior a $1 \%$ da carga horária integral), o reduzido número de docentes com formação em bioética, além do conteúdo oferecido em discussões teóricas nos anos intermediários da graduação e a inserção não longitudinal, como seria o ideal ${ }^{14-17}$.

Com o objetivo de conhecer a importância atribuída às disciplinas de Ética Médica, Bioética e Cuidados Paliativos na graduação médica, a carga horária $(\mathrm{CH})$ e a localização semestral e anual, foram analisados os dados obtidos nos sítios eletrônicos ${ }^{10,11}$ das $28 \mathrm{EM}$ distribuídas por regiões geoeconômicas do Estado de Minas Gerais. Os registros dessas EM foram estudados utilizando-se a metodologia de revisão integrativa, com as palavras-chave: ética médica, bioética, deontologia, medicina legal e $\mathrm{CP}$, para se conhecer a realidade do ensino de ética no curso de graduação em Medicina.

A maioria das informações era concisa quanto à metodologia aplicada, mas algumas informações não eram claras devido à ampla terminologia curricular, e não se teve acesso às ementas dessas disciplinas. Assim, uma parte da análise baseou-se na grade curricular, o que pode ter ocasionado interpretação incorreta dos dados coletados.

A análise documental do ensino de Ética nas 28 EMMG (Tabela 1) mostrou que estas escolas possuem a disciplina de Ética

\begin{tabular}{|c|c|}
\hline \multicolumn{2}{|l|}{$\begin{array}{c}\text { TABELA } 1 \\
\text { Sinopse do ensino de ética nas escolas } \\
\text { médicas de Minas Gerais }\end{array}$} \\
\hline Disciplina & $\begin{array}{l}\text { No de } \\
\text { escolas } \\
\text { médicas }\end{array}$ \\
\hline $\begin{array}{l}\text { Bioética; Introdução à Bioética; Ética e Bioética; } \\
\text { Medicina Legal/Bioética }\end{array}$ & 13 \\
\hline $\begin{array}{l}\text { Deontologia; Deontologia Médica; Medicina Legal e } \\
\text { Deontologia; Medicina Legal e Deontologia Médica; } \\
\text { Desenvolvimento Pessoal II (Deontologia Médica) }\end{array}$ & 6 \\
\hline $\begin{array}{l}\text { Ontoética: Ética e Moral; Ética; Ética e Bioética; } \\
\text { Ética e Espiritualidade; Ética e Bioética I, II, III; } \\
\text { Ética e Humanismo I, II, III; Ética Médica; Ética } \\
\text { no Início da Vida; Ética, Bioética e Saúde Pública; } \\
\text { Introdução à Ética Médica; Filosofia: Antropologia } \\
\text { e Ética; Psicologia Médica e Ética; Antropologia e } \\
\text { Sociologia Aplicada à Saúde; Antropologia Médica; } \\
\text { Antropologia Social }\end{array}$ & 15 \\
\hline
\end{tabular}

Medicina Legal; Medicina Legal e Deontologia;

Medicina Legal e Deontologia Médica; Medicina

Legal I; Medicina Legal/Bioética; Medicina Legal/

Ética Médica I, II; Atenção à Saúde VII (Medicina

Legal)

Psicologia Geral; Psicologia I, II; Psicologia Médica;

Psicologia Médica I, II; Psicologia Médica Aplicada;

Psicologia Médica e Ética; Psicologia Médica:

Abordagem Inicial do Paciente; Bases Psicossociais da Prática Médica

Tanatologia e Cuidados Paliativos I, II, III

Fonte: Dados da pesquisa. 


\begin{tabular}{|c|c|c|c|}
\hline \multicolumn{4}{|c|}{$\begin{array}{r}\text { QUADRO } 2 \\
\text { Respostas dos questionários aplicados aos alunos do }\end{array}$} \\
\hline \multicolumn{2}{|c|}{ Categorias/Unidades temáticas } & $\begin{array}{l}\text { Característica da EM } \\
\text { (número de alunos) }\end{array}$ & Professor \\
\hline Enfoque teórico & Enfoque teórico & $\begin{array}{l}\text { Tradicional (0) } \\
\text { Inovadora (4) } \\
\text { Avançada (1) }\end{array}$ & Inovadora \\
\hline Abordagem pedagógica & Estrutura curricular & $\begin{array}{l}\text { Tradicional (4) } \\
\text { Inovadora (1) } \\
\text { Avançada (0) }\end{array}$ & Tradicional \\
\hline $\begin{array}{l}\text { Abordagem em Ética e } \\
\text { Bioética }\end{array}$ & Disciplinas de Ética & $\begin{array}{l}\text { Ética Legal (5) } \\
\text { Ética Legal/Bioética (0) }\end{array}$ & Ética Legal \\
\hline \multirow{2}{*}{$\begin{array}{l}\text { Abordagem em } \\
\text { Cuidados Paliativos }\end{array}$} & $\begin{array}{l}\text { Referenciais teóricos em } \\
\text { Bioética }\end{array}$ & Bioética Clínica (5) & Bioética Clínica \\
\hline & $\begin{array}{l}\text { Concepção e disciplina de } \\
\text { Cuidados Paliativos }\end{array}$ & $\begin{array}{l}\text { Bioética (0) } \\
\text { Bioética / CP (0) } \\
\text { Cuidados Paliativos (0) }\end{array}$ & $\begin{array}{l}\text { Não possui departamento universitário e/ } \\
\text { ou corpo docente especializado em Medicina } \\
\text { Paliativa }\end{array}$ \\
\hline
\end{tabular}

Fonte: Dados da pesquisa.

e/ou conteúdo correlato em outras disciplinas curriculares, com níveis e abordagens diversos. Anteriormente, Ribeiro e Julio ${ }^{18}$ analisaram 18 matrizes curriculares de 26 EMMG, tendo encontrado resultado menos otimista: seis não possuíam disciplina específica sobre ética, bioética, deontologia ou responsabilidade civil. Dos 12 cursos restantes, nove oferecem a disciplina Ética, oito a disciplina Bioética, e apenas três a disciplina Deontologia ${ }^{18}$.

A análise comparativa dos dois estudos demonstra que houve mudanças num intervalo de quatro anos do ensino da ética nas EMMG. A disciplina Ética aparece com nomes consagrados, como Deontologia Médica, Medicina Legal, Ética Médica, e outros inovadores, como Antropologia e Ética, Psicologia Médica e Ética, Filosofia e Ética, Ética e Espiritualidade, Ética e Humanismo, Ética do Início da Vida, Ética e Bioética, e neologismos, como Ontoética. Já a disciplina Bioética é oferecida por 13 EMMG, correspondendo a 46,5\% das EM pesquisadas, mas a carga horária é reduzida (na faixa de 20 a 40 horas), e o conteúdo é introduzido nos anos iniciais e intermediários de graduação. Uma EM possui disciplina de Cuidados Paliativos.

As disposições curriculares das EMMG, com ênfase em bioética e em $\mathrm{CP}$, foram bem variadas, tanto na análise dos dados primários coletados (respostas aos questionários), quanto na análise dos dados secundários (registros eletrônicos de matrizes curriculares). Há algumas interrogações: se a Bioética deve ser uma nova disciplina ou mesmo substituir a disciplina Ética Médica e se pode prescindir da criação de disciplinas formais nessas áreas do conhecimento ${ }^{19,20}$. Rego ${ }^{20}$ diz que estamos longe de possuir docente competente, tanto no domínio da bioética, quanto de seu papel no processo de desenvolvi- mento moral de alunos, o que não permite a implantação de mudanças no ensino curricular e transversal de bioética.

\section{ANÁLISE DOS QUESTIONÁRIOS E ENTREVISTAS DA EM1}

Os alunos do sexto ano avaliaram a EM1 como tradicional e inovadora, enquanto o professor a qualificou como inovadora no enfoque teórico. Há discordância entre os alunos e o professor na abordagem pedagógica e na estrutura curricular, apontadas como de grau inovador pelos discentes e de grau tradicional pelo docente. A análise apresentou resultados equilibrados (alunos e professor) nas abordagens de ética e bioética, com a referência da disciplina Medicina Legal e abordagem de bioética. Ela não possui disciplina de CP (Quadro 2).

A seguir, extraímos alguns depoimentos obtidos dos questionários aplicados na EM1 para ilustrar a pertinência da reflexão do professor e dos estudantes sobre os seus processos de aquisição e construção do conhecimento científico.

A dicotomia curricular entre o ciclo básico e o ciclo clínico dificulta o aprendizado de forma crescente ou espiralar, como se percebe:

Há uma dicotomia entre o ciclo básico e o profissionalizante, organizado em disciplinas modulares específicas. (professor)

A proposta pedagógica do curso aproxima-se da alternativa referente ao currículo integrado. Entretanto, existe delimitação dos ciclos básico e profissionalizante, e as matérias modulares nem sempre alcançam o inter-relacionamento ao qual se propõem. (aluna) 
Ciclo básico muito descontextualizado. Não há correlação com a prática médica no ciclo básico. (aluna)

O professor da EM1 afirma que as mudanças curriculares e a implementação de práticas integrativas estão em pauta na perspectiva de reduzir as distâncias entre os segmentos polares do ensino e da aprendizagem.

As dificuldades encontradas pelos professores e suas consequências para os estudantes no ensino-aprendizagem de ética e de bioética podem ser vistas nos depoimentos dessas estudantes:

Não há referenciais teóricos em abordagem ou estudo aprofundado, embora se discutam problemáticas de saúde e prática profissional em sua interface com a ética. (aluna)

Alguns temas sobre ética e bioética foram discutidos durante a disciplina Tutoria (quinto período) de acordo com a demanda dos alunos. Foi uma abordagem reflexiva, sem enfocar referenciais teóricos ou seguindo roteiro prévio. (aluna)

A formação do corpo docente relativa aos temas de ética e bioética mereceu comentários de alunas do terceiro ano preocupadas com uma formação profissional humanista, crítica e reflexiva, justificada em princípios éticos, legais e bioéticos ${ }^{21}$.

Alguns professores abordam os referenciais bioéticos, desenvolvem reflexões e discutem problemas, porém eles são minoria. (aluna)

As discussões são superficiais, muitas vezes apenas para explicar o porque de se adotar ou não uma dada prática diante do paciente. "Não seria ético falar isso" na frente do paciente. (aluna)

Continuando a coleta de dados (terceira etapa), uma entrevista estruturada foi aplicada a um grupo de cinco alunos da EM1 que cursavam o sexto ano (11ํ e $12^{\circ}$ períodos), especificamente do internato de Clínica Médica no HE, objetivando responder às seguintes perguntas: $\mathrm{O}$ curso de Medicina da EM1 possui, na grade curricular, a disciplina de Bioética e Cuidados Paliativos? Além do conteúdo teórico desenvolvido em sala de aula de outras disciplinas, você observa que os princípios bioéticos também são aplicados na prática do HE? Você já presenciou situações em que o professor ou médico-residente discute esse tema diante de um paciente em fase terminal de doença?

A seguir, citam-se os depoimentos de alunos do sexto ano da EM1 sobre estas questões:
Apesar de não apresentar na grade curricular disciplina específica de Bioética e Cuidados Paliativos, o curso de Medicina possibilita refletir, por diversas vezes, sobre esse assunto, principalmente em seu ciclo clínico. Acompanhar discussão de caso com os serviços de Clínica Médica, Oncologia e Clínica de Dor aumentou ainda mais tal possibilidade. (aluna)

O HE é um hospital de alta complexidade e referência para Oncologia, ocorrendo grandes discussões sobre os pacientes com doenças graves. (aluno)

Os relatos mostram que a ausência curricular de Bioética e Cuidados Paliativos não dificultou a reflexão bioética frente ao paciente com doença avançada. Mas uma discussão de caso não envolve necessariamente uma reflexão bioética. Pode ser meramente uma discussão baseada em fatos, não focando a análise e reflexão de valores morais. A lacuna curricular é suprida, em parte, pelo trabalho da equipe de Cuidados $\mathrm{Pa}$ liativos no HE, difundindo o conhecimento e a reflexão neste campo emergente da prática médica.

No depoimento de alunos entrevistados com o ensino-aprendizagem médico internacional ${ }^{22,23}$, há correlação quanto às questões relativas à abordagem pedagógica sobre $\mathrm{CP}$. Um aluno sugere a inserção da disciplina sobre $\mathrm{CP}$ no currículo da EM1.

O princípio bioético é aplicado ou não é aplicado de acordo com o conhecimento do professor e/ou médico-residente ou a importância que ele dá à bioética. (aluno)

Os pacientes do HE têm um perfil de doenças mais raras $e$, muitas vezes, evoluem rapidamente para os cuidados paliativos, o que nos propicia, diariamente, presenciar essas situações. (aluna)

Depende do professor e/ou do médico-residente. Eu vejo que o ensino dessas áreas é muito restrito e já presenciei, várias vezes, situações tanto de posturas éticas quanto de total desrespeito para com o paciente. (aluna)

[...] mas acredito que se deveriam formalizar as disciplinas curriculares (Bioética e Cuidados Paliativos). (aluno)

\section{ANÁLISE DOS QUESTIONÁRIOS E ENTREVISTAS DA EM2}

No enfoque teórico, os alunos avaliaram a EM2 como tradicional e inovadora, e o professor a qualificou como inovadora. Já na abordagem pedagógica e estrutura curricular, há discordância, pois foi apontada como inovadora pelos alunos e tradicional pelo professor. A análise apresentou resultados equilibrados entre as abordagens de ética, bioética e CP (Quadro 3). 


\begin{tabular}{|c|c|c|c|}
\hline \multicolumn{4}{|c|}{$\begin{array}{c}\text { QUADRO } 3 \\
\text { Respostas dos questionários aplicados aos alunos } \\
\text { do sexto ano e professor da Escola Médica } 2\end{array}$} \\
\hline \multicolumn{2}{|c|}{ Categorias e unidades temáticas } & $\begin{array}{c}\text { Tipologia } \\
\text { da FM / } \\
\text { (no de alunos) }\end{array}$ & Professor \\
\hline $\begin{array}{l}\text { Enfoque } \\
\text { teórico }\end{array}$ & Enfoque teórico & $\begin{array}{l}\text { Tradicional (2) } \\
\text { Inovadora (3) } \\
\text { Avançada (0) }\end{array}$ & Inovadora \\
\hline \multirow[b]{2}{*}{$\begin{array}{l}\text { Abordagem } \\
\text { pedagógica }\end{array}$} & $\begin{array}{l}\text { Estrutura } \\
\text { curricular }\end{array}$ & $\begin{array}{l}\text { Tradicional (1) } \\
\text { Inovadora (4) } \\
\text { Avançada (0) }\end{array}$ & Tradicional \\
\hline & Conteúdos & $\begin{array}{l}\text { Tradicional (0) } \\
\text { Inovadora (2) } \\
\text { Avançada (3) }\end{array}$ & $\begin{array}{l}\text { Avançada } \\
\text { (disciplina } \\
\text { humanística } \\
\text { e capacitação } \\
\text { docente) }\end{array}$ \\
\hline $\begin{array}{l}\text { Abordagem } \\
\text { em Ética e } \\
\text { Bioética }\end{array}$ & $\begin{array}{l}\text { Disciplinas de } \\
\text { Ética }\end{array}$ & $\begin{array}{l}\text { Ética Legal (0) } \\
\text { Ética Legal/ } \\
\text { Bioética (2) } \\
\text { Bioética/ } \\
\text { transversal (2) } \\
\text { Nula (1) } \\
\end{array}$ & $\begin{array}{l}\text { Ética Legal } \\
\text { Bioética: disciplina } \\
\text { e transversal }\end{array}$ \\
\hline \multirow{2}{*}{$\begin{array}{l}\text { Abordagem } \\
\text { em Cuidados } \\
\text { Paliativos }\end{array}$} & $\begin{array}{l}\text { Referenciais } \\
\text { teóricos em } \\
\text { Bioética }\end{array}$ & $\begin{array}{l}\text { Nula (1) } \\
\text { Bioética Clínica (4) }\end{array}$ & Bioética Clínica \\
\hline & $\begin{array}{l}\text { Concepção } \\
\text { e disciplina } \\
\text { de Cuidados } \\
\text { Paliativos }\end{array}$ & $\begin{array}{l}\text { Nula }(1) \\
\text { Bioética }(0) \\
\text { Bioética/CP (1) } \\
\mathrm{CP} / \text { transversal (3) }\end{array}$ & $\begin{array}{l}\text { Possui disciplinas } \\
\text { de Tanatologia } \\
\text { e Cuidados } \\
\text { Paliativos }\end{array}$ \\
\hline
\end{tabular}

Nota: Resposta "Nula" refere-se a "Não respondeu" (não disponibilizou o dado). Fonte: Dados da pesquisa.

Continuando a coleta de dados (terceira etapa), quatro alunos foram novamente entrevistados durante o internato hospitalar, com as seguintes perguntas: Além do conteúdo teórico desenvolvido em sala de aula, você observa que os princípios bioéticos também são aplicados na prática do HE? Você já presenciou situações em que o professorou médico-residente discute esse tema diante de um paciente em fase terminal de doença?

Alguns depoimentos extraídos das respostas dos alunos da EM2 ilustram a reflexão de estudantes sobre seus processos de aquisição e prática do conhecimento de CP:

Durante a graduação, muitos professores abordaram este tema em sala de aula, com a finalidade de nos tornarmos profissionais mais humanizados, buscando sempre prevenir e aliviar o sofrimento dos pacientes com doença progressiva e irreversível, promovendo sua melhor qualidade de vida e de sua família. Porém, durante a prática médica na enfermaria do HE, tais princípios não são discutidos com a mesma intensidade diante da doença de cada paciente, como ocorria em sala de aula. (aluna)
Mas essa discussão [sobre a qualidade de vida dos pacientes terminais] não é levada como cuidados paliativos. Muitas vezes, parece que cuidado paliativo é uma especialidade da Medicina, que tem que ser estudada para ser aplicada, e não uma área médica que naturalmente está incluída no dia a dia da enfermaria. (aluna)

Ayre ${ }^{24}$ comenta as diferenças entre o currículo formal e as experiências clínicas em campo nos estágios no HE. O autor adverte que, além de haver a disciplina Medicina e Humanidades Médicas, é preciso "[...] interferir na cultura institucional do Hospital das Clínicas e nos demais serviços em que ocorre aprendizado, de modo a que eles (alunos) percebam a busca do cuidado como um valor compartilhado."24 (p. 141).

A análise comparativa dos resultados de questionários aplicados aos alunos do último ano do curso médico de EM1 e EM2 mostrou uma aparente semelhança. Mas foi apenas um equilíbrio aparente, o que está de acordo com os dados secundários de autores que foram introduzidos na construção do objeto e na triangulação metodológica deste estudo. Por isso, foi necessário entrevistar em profundidade os alunos, em especial aqueles da EM1, sobre a formação curricular e a vivência à beira do leito frente às questões éticas da terminalidade da vida humana. Estas entrevistas comprovam a necessidade de uma formação direcionada para essa temática.

A fundamentação de um modelo teórico e prático do ensino de bioética e de CP necessita de reflexão, além de se discutir nas EMMG o preparo do docente no tocante a sua formação e competência em bioética e em CP.

\section{DISCURSO PROGRAMÁTICO}

O campo das humanidades visto na Sociologia Médica ${ }^{24,25,26}$ deveria estar presente de forma longitudinal na formação do futuro médico, de forma humanista, crítica e reflexiva, para contribuir na formação sociológica e antropológica do médico, "[...] como condição para um entendimento do homem; não só do homem doente, mas das circunstâncias que envolvem o adoecimento. ${ }^{26}$ (p. 13). O treinamento de algumas habilidades e atitudes, como empatia, reflexão ética e bioética, comunicação e deliberação, deveria inserir-se no currículo das EM e contribuir para a formação qualificada dos futuros profissionais, tanto na competência técnica como na competência humanística. A respeito do ensino de humanidades médicas, há necessidade de ampliar os existentes, inserir outros e valorizar todos, abrindo frestas para a implantação efetiva de $\mathrm{CP}$ no contexto teórico e prático das EM.

A atitude do homem diante do processo do morrer e da morte, na contemporaneidade, não será equacionada, consi- 
derando o aspecto curricular vigente na maioria das EM, que objetiva a cura anatômica das doenças. De certa forma, o paradigma hegemônico do modelo de ensino médico (tipologia tradicional) - demarcado pela estrutura curricular fracionada de ensino-aprendizagem em aulas teóricas expositivas, práticas demonstrativas com grupo grande de alunos e um método clínico que não considera a individualidade do sujeito - constitui uma barreira à mobilização de docentes e discentes frente à complexa educação do viver e do morrer.

Há questões coexistentes ao ensino de farmacologia e dos opioides. É fato que a farmacologia é ministrada no ciclo básico do curso médico, distante do enfoque voltado para a prática médica atual. É necessário informar os alunos sobre a estatística de consumo per capita de opioides como um indicador de qualidade de saúde pública mundial e a restrição paradoxal no Brasil.

Para a formação do futuro médico, é essencial desenvolver habilidades cognitivas, psicomotoras e atitudinais contextualizadas à prática médica atual. O cuidado - cuidar do outro - no campo da saúde é um dos pressupostos da dignidade humana.

No processo do morrer e da morte, o sujeito precisa de solidariedade e de reconhecimento de sua dignidade, e não viver preso a máquinas, fios e tubos, longe do contato humano ${ }^{8,27}$. Todavia, para que não ocorra esse encarceramento quase mecânico do paciente, é necessário que o ensino médico se volte também para despertar a sensibilidade de educadores e estudantes, "[...] para o cuidado do paciente terminal, buscando uma formação interdisciplinar dos médicos." ${ }^{\prime 8}$ (p. 288). A interdisciplinaridade no campo de humanidades médicas, integrando as disciplinas ou módulos curriculares, mobilizaria a capacitação docente para outras áreas do conhecimento, o que tornaria a prática pedagógica mais apta a integrar o discurso interdisciplinar sobre o desenvolvimento humano (ciclos de vida).

Entretanto, docentes da USP afirmam que "[...] o ensino de temas das áreas humanas para a construção da identidade e atitude médica, aqui e em vários lugares no mundo, é tarefa difícil que, somada a problemas estruturais, encontra resistências em alunos e professores." ${ }^{25}$ (p. 113). A avaliação do ambiente de ensino poderia identificar aspectos da educação médica e necessidades curriculares que, se investigados, possibilitariam verificar os pontos de resistência às mudanças e propor medidas para superá-los ${ }^{28}$.

\section{REFLEXÕES E CONCLUSÕES}

Como problemática, foram identificadas questões como: desinteresse de professores da área médica em introduzir a disciplina de Cuidados Paliativos na grade curricular; falta de evidência nas propostas pedagógicas, implantando disciplina sobre a temática do cuidado com paciente portador de doença terminal, quando esses educadores almejam mudanças curriculares. É possível que o motivo do desinteresse e da falta de evidência para inserir esta disciplina nos currículos das EMMG esteja ligado a certo temor desses profissionais de enfrentarem a dramática questão da terminalidade da vida humana.

Os resultados mostraram que os professores, responsáveis pela elaboração dos currículos, não se preocupam com a disseminação do conhecimento nas áreas de bioética com ênfase em CP, com a formação de médicos que atendam às necessidades emergentes desta área da ciência e com a articulação entre ensino de bioética e CP.

A discussão de temas relacionados à bioética e ao $\mathrm{CP}$ por parte do corpo de professores do HE poderia modificar o comportamento dos médicos, tornando-os aptos a lidar com o paciente com doença avançada e terminal. Logo, a educação formal (disciplinas) e a informal (ligas acadêmicas), a esse respeito, podem implementar nos alunos habilidades e atitudes éticas, perspicácia, prudência, virtude, desde o início da graduação.

O processo de integração de Ética Médica, Bioética e Deontologia Médica encontra-se em expansão curricular na graduação das EM do Brasil. Também houve mudanças em Minas Gerais. As competências em bioética são complexas e necessárias à formação do futuro médico para terem, ainda, tão reduzido espaço no currículo formal. Com carência ainda maior, constata-se uma ausência da disciplina, obrigatória ou optativa, de Cuidados Paliativos nas EMMG, tendo como pré-requisito a disciplina de Bioética.

É necessária a inserção do CP nos cursos de Medicina, seja no formato de disciplina específica, em curto prazo, seja por meio de um ensino longitudinal, em longo prazo, quando poderá ser discutido durante a graduação, em outra disciplina ou módulo curricular. Esta inserção acadêmica, assim como da Bioética, é fundamental à consolidação do CP nas EMMG e, possivelmente, à regionalização, nas EM do Brasil.

A unicidade do tema demanda outros trabalhos acadêmicos e maior participação da sociedade à qual o cuidado é direcionado. A sociedade laica pode e deve participar desta discussão. Da mesma forma, a divulgação midiática deste tema deve mobilizar uma reflexão crítica da comunidade acadêmica e promover uma abertura na agenda dos profissionais, gestores e professores responsáveis pela elaboração curricular e pelo projeto político-pedagógico nas EMMG e do Brasil, para se discutir e efetivar a inserção de cuidados paliativos. 


\section{REFERÊNCIAS}

1. Antonello ICF. Cuidando de cuidadores em formação nas faculdades de medicina. Revista Bioética 2006;14(2):15962.

2. Brasil. Ministério da Saúde. Departamento de Informática do SUS. Indicadores e Dados Básicos - Brasil - 2006. Brasília: Ministério da Saúde, 2006. [online]. Disponível em:<http://tabnet.datasus.gov.br/cgi/idb2006/matriz. $\mathrm{htm}>$. Acesso em: 12 dez. 2012.

3. Wagner D. Estado da arte dos cuidados paliativos oncológicos: retrato da assistência no município de Juiz de Fora. Juiz de Fora; 2013. 175f. Mestrado [Dissertação] -Universidade Federal de Juiz de Fora.

4. Silva RCF, Hortale VA. Cuidados paliativos oncológicos: elementos para o debate de diretrizes nesta área. Cad Saúde Pública 2006;22(10):2055-66.

5. Garrett TM, Baillie HW, Garrett RM. Health care ethics: principles and problems. Englewood Cliffs NJ: Prentice Hall; 1989. 271p.

6. OliveiraJR, Amaral CFS, Ferreira AC, Grossi YS, Rezende NA. Percepção bioética sobre a dignidade no processo de morrer. Revista Bioética 2009;17(1):77-94.

7. Pinheiro T, Blasco P, Benedetto MA, Levites M, Giglio AD, Monaco $\mathrm{C}$. Teaching palliative care in a free clinic: a Brazilian experience. In: Chang E, Johnson A. Contemporary and innovative practice in palliative care. Croatia: InTech; 2012. 302p. Disponívelem: <http://cdn.intechopen.com/ pdfs/27611/ InTech-Teaching_palliative_care_in_a_free_ clinic_a_Brazilian_experience.pdf $>$. Acesso em: 17dez. 2015.

8. Oliveira JR, Ferreira AC, Rezende NA. Ensino de bioética e cuidados paliativos nas escolas médicas do Brasil. Rev Bras Educ Med 2013; 37(2):285-90.

9. Academia Nacional de Cuidados Paliativos. O Congresso: Notícias, IV Congresso Internacional de Cuidados Paliativos, 6-9 out. 2010, São Paulo: ANCP, 25 jul. 2010. [13 telas]. Disponível em: <http:/ / www.paliativo.org.br/congresso/noticias/tag/the-economist/>. Acesso em: 18 jan. 2013.

10. Nassif ACN. Escolas Médicas do Brasil. São Paulo: Nassif; 2014. Disponível em: <http://www.escolasmedicas.com. br/estat.php>. Acesso em: 19 dez. 2014.

11. Brasil. Ministério da Educação. Sistema e-MEC. Instituições de Educação Superior e Cursos Cadastrados. Brasília: MEC; 2014. Disponível em: <http://emec.mec.gov.br/>. Acesso em: 23 jan. 2014.

12. Lampert JB. Tendências de mudanças na formação médica no Brasil. São Paulo: HUCITEC; 2002. 283p.
13. Finkler M. Formação ética em Odontologia: realidades e desafios. Florianópolis, 2009. Doutorado [Tese] - Universidade Federal de Santa Catarina.

14. Muñoz D, Muñoz DR. O ensino da ética nas Faculdades de Medicina do Brasil. Rev Bras Educ Med. 2003;27(2):114-24.

15. Dantas F, Sousa EG. Ensino da Deontologia, Ética Médica e Bioética nas Escolas Médicas Brasileiras: uma revisão sistemática. Rev Bras Educ Med. 2008;32(4):507-17.

16. Guedert JM. Vivência de problemas éticos em pediatria e sua interface com a educação médica. Florianópolis, 2012. Doutorado [Tese] - Universidade Federal de Santa Catarina.

17. Camargo A, Almeida MAS, Morita I. Ética e Bioética - o que os alunos do $6^{\circ}$ ano médico têm a dizer. Rev Bras Educ Med, 2014;38(2):182-9.

18. Ribeiro WC, Julio RS. Reflexões sobre erro e educação médica em Minas Gerais. Rev Bras Educ Med 2011; 35(2):2637.

19. Costa NMSC. A formação e as práticas educativas de professores de medicina: uma abordagem etnográfica. São Paulo; 2005. Doutorado [Tese] - Pontifícia Universidade Católica de São Paulo.

20. Rego S. Reflexão sobre o processo de formação ética dos médicos. Cadernos da ABEM. 2004; 1(1):28-9.

21. Brasil. Ministério da Educação. Conselho Nacional de Educação. Câmara de Educação Superior. Resolução CNE-CES n. 4, de 7 de novembro de 2001. Institui as Diretrizes Curriculares Nacionais do Curso de Graduação em Medicina. Diário Oficial da União, 9 nov. 2001, Seção 1, p. 38.

22. Billings JA, Block SD. Palliative care in undergraduate medical education: status report and future directions. Journal American Medicine Association 1997; 278:733-8.

23. BillingsME, Engelberg R, Curtis JR, Block S, Sullivan AM. Determinants of medical students' perceived preparation to perform end-of-life care, quality of end-of-life care education, and attitudes toward end-of-life care. Journal of Palliative Medicine 2010;13(3):319-26.

24. 24. Ayres JRCM. In: Tostes MA. (Org.) (Des)encontro do médico com o paciente: o que pensam os médicos? Rio de Janeiro: Rubio; 2014. 346p.

25. Rios IC, Lopes Junior A, Kaufman A, Vieira JE, Scanavino MT, Oliveira, RA. A integração das disciplinas de humanidades médicas na faculdade de medicina da USP: um caminho para o ensino. Rev Bras Educ Med 2008;32(1):11221.

26. Rasia JM. Prefácio à presente edição. In: Freyre G. Sociologia da Medicina. 3. ed. São Paulo: É Realizações; 2009. 288p. 
27. Pessini L, Bertachini L. (Org.). Humanização e Cuidados Paliativos. 2. ed. São Paulo: Loyola; 2004. 319p.

28. Vieira JE, Tamousauskas MRG. Avaliação das resistências de docentes a propostas de renovações em currículos de graduação em medicina. Rev Bras Educ Med. 2013;37(1):32-8.

\section{CONTRIBUIÇÃO DOS AUTORES}

José Ricardo de Oliveira contribuiu na concepção e desenho do estudo, análise e interpretação dos dados da pesquisa, produção e revisão do texto final. Nilton Alves de Rezende e Amauri Carlos Ferreira participaram da concepção do estudo e revisão do texto final. Letícia Pereira de Castro participou da coleta e interpretação dos dados e revisão do texto final.

\section{CONFLITO DE INTERESSES}

Os autores declaram não haver conflito de interesses.

ENDEREÇO PARA CORRESPONDÊNCIA

José Ricardo de Oliveira

Rua Maracaju, 19 - apto 1603

Nova Granada - Belo Horizonte

CEP 30431-350 - MG

E-mail: oliveira.jricardo@gmail.com 